\title{
Activation of the JNK pathway during dorsal closure in Drosophila requires the mixed lineage kinase, slipper
}

\author{
Beth Stronach and Norbert Perrimon ${ }^{1}$ \\ Department of Genetics, Howard Hughes Medical Institute, Harvard Medical School, Boston, Massachusetts 02115, USA
}

\begin{abstract}
The Jun kinase (JNK) pathway has been characterized for its role in stimulating AP-1 activity and for modulating the balance between cell growth and death during development, inflammation, and cancer. Six families of mammalian kinases acting at the level of JNKKK have emerged as upstream regulators of JNK activity (MLK, LZK, TAK, ASK, MEKK, and TPL); however, the specificity underlying which kinase is utilized for transducing a distinct signal is poorly understood. In Drosophila, JNK signaling plays a central role in dorsal closure, controlling cell fate and cell sheet morphogenesis during embryogenesis. Notably, in the fly genome, there are single homologs of each of the mammalian JNKKK families. Here, we identify mutations in one of those, a mixed lineage kinase, named slipper (slpr), and show that it is required for JNK activation during dorsal closure. Furthermore, our results show that other putative JNKKKs cannot compensate for the loss of slpr function and, thus, may regulate other JNK or MAPK-dependent processes.
\end{abstract}

[Key Words: JNK; morphogenesis; dorsal closure; kinase]

Received October 11, 2001; revised version accepted December 5, 2001.

Eukaryotes transduce a variety of signals by use of a well-conserved kinase phosphorylation cascade culminating in activation of a class of proteins, generically referred to as mitogen-activated protein kinases (MAPKs) (Chang and Karin 2001). Typically, activated MAPKs phosphorylate transcription factor substrates, thus, ultimately regulating gene expression and cellular behavior. MAPKs are the most downstream kinases in a tripartite module of protein kinases. As such, MAPKs are phosphorylated and activated by dual specific MAPK kinases (MAPKKs/MKKs), which are themselves phosphorylated and activated by another family of upstream serine/threonine kinases, the MAPK kinase kinases (MAPKKKs, MKKKs).

Signals that stress cells during inflammation or changing environmental conditions induce a particular class of MAPKs called the stress-activated protein kinases (SAPKs). SAPKs are also referred to as JNKs because they phosphorylate the NH2 terminus of cJun, which together with cFos constitutes the AP-1 (activator protein1) transcriptional complex that regulates primary response genes (Davis 2000). As evidenced by the constitutive active nature of the viral v-jun and v-fos oncogenes described over a decade ago, proper regulation of AP-1 activity under varied conditions is critical for

${ }^{1}$ Corresponding author.

E-MAIL perrimon@rascal.med.harvard.edu; FAX (617) $432-7688$.

Article and publication are at http://www.genesdev.org/cgi/doi/10.1101/ gad.953002. normal cellular behavior (Vogt 2001). In certain cell types, simply overexpressing proteins that compose AP-1 or positively regulate its activity can lead to transformation (Hartkamp et al. 1999; Jochum et al. 2001) whereas, in other contexts, JNK stimulation can induce apoptosis (Ip and Davis 1998; Jochum et al. 2001). Given the diverse and often conflicting roles ascribed to JNK signal transduction, it is important to define how distinct extracellular signals are specifically coupled to the JNK module and thus to the proper transcriptional output via JNK targets. Furthermore, characterization of a growing number of mammalian kinases with the ability to stimulate JNK activation in cell assays highlights the complexity and potential lack of specificity among JNKKKs. Many questions remain, such as why are there so many distinct kinase families at this level of the signaling hierarchy? Perhaps each kinase family interprets a distinct upstream signal or participates in unique cellular and developmental processes. Loss-of-function analysis will be a crucial step in answering these questions.

Toward this end, we have taken a genetic approach to define regulators of the JNK pathway in vivo using Drosophila dorsal closure as a model system for JNK-dependent signal transduction (Knust 1997; Noselli 1998). In the fly embryo, dorsal closure involves cell sheet morphogenesis, whereby the dorsal ectoderm moves from an initial lateral position toward the dorsal midline to enclose the mature embryo in a continuous protective epidermis. Mutations in the fly homologs of the vertebrate members of the JNK signal transduction pathway, hemipterous (hep), a JNKK related to vertebrate MKK7 
(Glise et al. 1995; Holland et al. 1997), basket (bsk), a JNK (Riesgo-Escovar et al. 1996; Sluss et al. 1996), dJun (Hou et al. 1997; Kockel et al. 1997b; Riesgo-Escovar and Hafen 1997), and dFos, encoded by kayak (kay) (Zeitlinger et al. 1997) result in dorsal closure failure, and thus, embryonic lethality. Despite significant progress in defining gene functions required for JNK signaling and dorsal closure in general, there are still several obvious missing players in the signaling machinery. Here, we report the identification and phenotypic characterization of an additional regulator of JNK activity, named slipper (slpr). Cloning reveals that slpr encodes a member of the mixed lineage kinase (MLK) family of Jun kinase kinase kinases. Our analysis provides the first demonstration of an absolute requirement for MLKs in the regulation of JNK signaling during morphogenesis in vivo and establishes a specific genetic link between MLK, MKK7, and JNK.

\section{Results}

Genetic characterization of slpr, a dorsal open mutant

Several large-scale genetic screens were undertaken in Drosophila to identify the maternal effects of zygotic lethal loci using the dominant female sterile technique (Perrimon et al. 1989; Chou and Perrimon 1996). Among the collection of mutants, we searched for those mutations that caused dorsal open embryonic cuticles indicative of a failure in the process of dorsal closure. On the basis of that cuticle phenotype, we identified several Xlinked mutations that produce consistent, severe, open cuticles when derived from females with mutant germline clones (Fig. 1A). Two mutations, 3P5 and 921, map to the same genetic region (see below). Later, we found that these mutations define the same gene that we call slpr, because the cuticle phenotype resembles an open shoe.

The dorsal open cuticle phenotype of slpr mutant embryos resembles that caused by mutations in components of the JNK pathway known to regulate the progression of dorsal closure and expression of genes in the leading edge of the epidermis (Glise and Noselli 1997; Hou et al. 1997; Kockel et al. 1997a; Riesgo-Escovar and Hafen 1997). To determine whether loss of slpr function affects JNK transcriptional targets, we characterized the expression of decapentaplegic $(d p p)$ in slpr mutant embryos. We found that $d p p$ expression is absent from leading edge cells of approximately one-quarter of the observed embryos (Fig. 1B), consistent with the expected fre-
Figure 1. Phenotypic effects of slpr mutations. (A) Embryonic cuticle phenotype of a strong loss-of-function slpr allele, 921, shows a large anterior dorsal hole compared with wild type (WT). (B) In situ hybridization to reveal $d p p$ transcripts in wild-type $\left(s \operatorname{spr}^{921} /+\right)$ or mutant $\left(\operatorname{slpr}^{921} / Y\right)$ embryos at stage 11, germ band extended, and stage 13 , germ band retracted. Stages according to Campos-Ortega and Hartenstein (1997). dpp expression is observed in cells at the dorsal edge of the ectoderm (arrowhead) in wild-type but not in slpr mutant embryos at both stages. Other aspects of $d p p$ expression are normal. (C) A collection of embryos from a slpr ${ }^{921} \mathrm{mu}-$ tant stock has been labeled with anti-Fasciclin III antibodies to reveal the shape of cells in the dorsal ectoderm. The bracket marks leading edge (LE) cells at the margin that have begun to stretch and elongate as dorsal closure proceeds in both wild-type $\left(s_{p r} r^{921} / F M 7, f t z L Z\right)$ and $s l p r^{921}$ mutant embryos. However, late stage $\operatorname{slpr}^{921} / Y$ mutant embryos exhibit a terminal phenotype in which LE cells fail to remain elongated and have rounded up, concomitant with slackening of the dorsal ectodermal sheet.
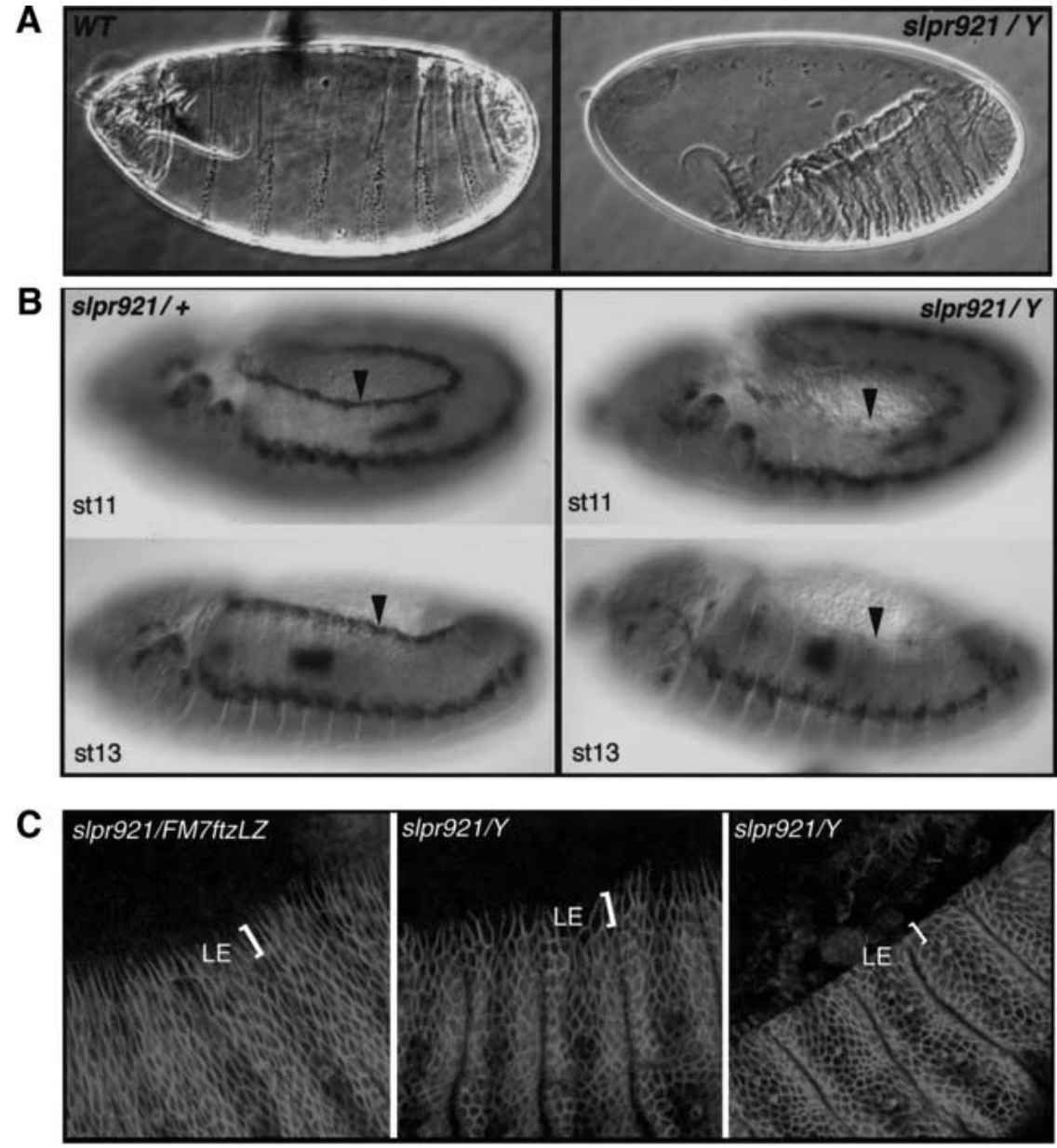
quency of zygotically mutant $s 1 p r^{921} / Y$ embryos. In the mutant embryos, the other tissue-specific patterns of $d p p$ expression are unaffected. Furthermore, nearly half of all embryos derived from females with germ-line clones of the $s 1 p r^{3 P 5}$ allele show loss of leading edge $d p p$ expression (data not shown). Thus, the specific loss of $d p p$ from leading edge cells of slpr mutant embryos indicates a significant reduction of JNK signaling and AP-1 activity.

The underlying defects that contribute to aborted dorsal closure in JNK pathway mutants are not only the failure to maintain leading edge specific gene expression but also the failure to maintain a stretched epithelial cell morphology (Glise et al. 1995; Riesgo-Escovar et al. 1996). Using a marker for epidermal cell boundaries, we found that slpr mutant embryos also do not maintain the dramatic cell shape changes within the dorsal epithelium (Fig. 1C). In particular, we observed that the concerted elongation of leading edge cells initiates properly but fails shortly afterward. Eventually, these cells round up, the dorsal epidermis slackens laterally, and the internal organs herniate. Because the cuticle is secreted from epidermal cells, and the dorsal epidermis has failed to close dorsally, the resulting cuticle has a large hole on the dorsal side.

The phenotypic similarities between slpr and genes encoding the JNK signaling cascade, hep, bsk, and dJun, suggest that slpr may regulate JNK signaling. To further test whether slpr mutants diminish signaling through the JNK pathway, genetic epistasis tests were performed. Activation of positive components functioning downstream of slpr may be expected to alleviate the defect caused by slpr loss-of-function. Inducible expression of a constitutive active form of the Jun transcription factor (Treier et al. 1995) that normally serves as a substrate for phosphorylation by Bsk significantly rescues the slpr mutant phenotype (Fig. 2A). Similarly, loss-of-function mutations in downstream negative components may augment residual signaling activity to functional levels.
Consistent with this line of reasoning, slpr is dominantly suppressed by reducing the dosage of a negative regulator of JNK signaling, puc, encoding a JNK phosphatase (Glise and Noselli 1997; Martin-Blanco et al. 1998). Heterozygosity at the puc locus significantly suppresses the severe cuticle phenotype of the strong slpr ${ }^{921}$ allele, indicated by the clear reduction in size of dorsal cuticle holes (Fig. 2B). Moreover, loss of one copy of puc rescues embryos mutant for the weaker $s_{p r^{3 P 5}}$ allele such that they develop to adulthood. Mutant male flies emerge but are weakly viable and show no gross morphological defects. Taken together, these data support a role for slpr in JNK signal transduction, upstream of bsk.

\section{Cloning reveals that slpr encodes a Jun kinase kinase kinase}

To identify the gene responsible for the slpr phenotype, we used several mapping strategies. Figure $3 \mathrm{~A}$ shows the genomic arrangement of markers and other aberrations used to position the slpr locus. Meiotic recombination with visible recessive markers and complementation mapping with large deletions positioned the slpr locus between 7D13 and 7E2 of the X chromosome. Additional recombination mapping between slpr and nearby P-element transposon insertions narrowed the region to 7D16-7D18. At this time, the Drosophila genomic DNA sequence became available (Adams et al. 2000). Having conservatively defined the outer limits demarcating the slpr locus to the area between Traf2 (7D13) and Trf2 (7E7), we noted a group of $\sim 30$ known and predicted loci as candidates for the slpr gene. Using gene-specific primers, we assayed for PCR amplification products from DNA templates derived from the deficiency lines. Only those loci that were absent from the deficiencies that remove slpr were considered further.

Among a restricted group of candidate genes that were present in $D f(1) H A 11$ DNA but not within $D f(1) G E 202$ DNA, we examined the predicted protein structure of

\section{A}

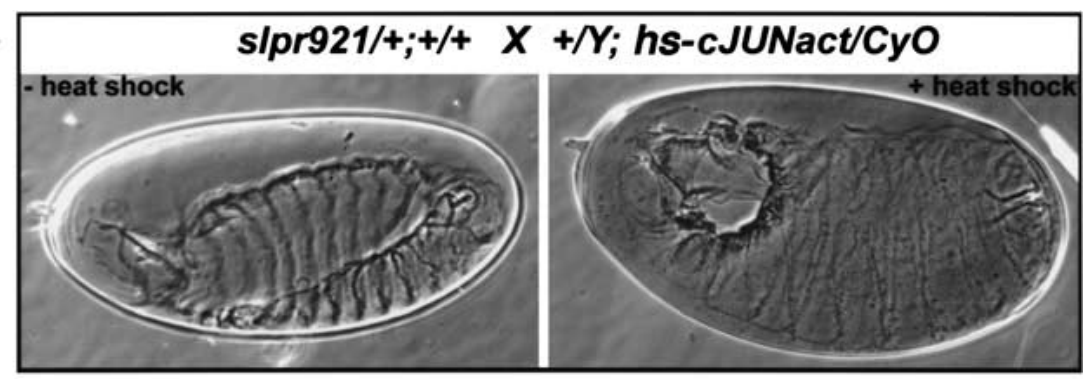

B

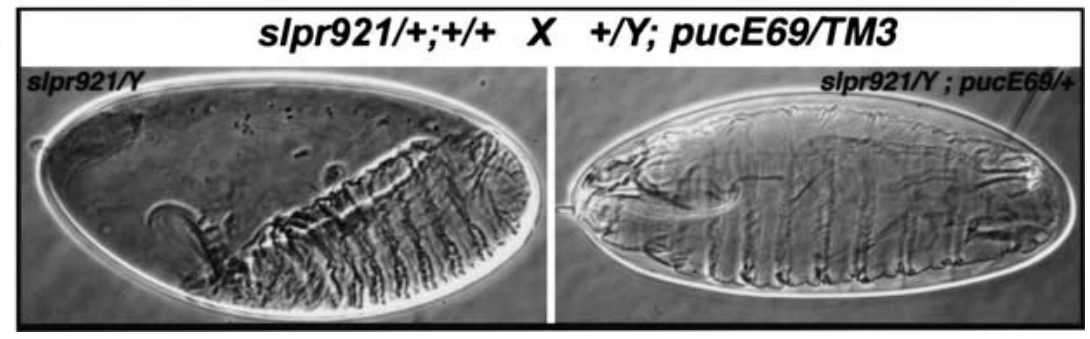

Figure 2. Epistasis analysis of slpr with JNK pathway components. (A) Embryonic cuticle preparation of progeny derived from the cross indicated and either maintained at $25^{\circ} \mathrm{C}$ (- heat shock) or subjected to a 30 min regimen at $37^{\circ} \mathrm{C}$ (+ heat shock) several hours prior to the beginning of dorsal closure. Embryos receiving heat shock express an inducible, constitutive active form of the cJun transcription factor that partially rescues the severe dorsal open phenotype of slpr ${ }^{921}$ mutant embryos. (B) Embryonic cuticle preparation of progeny derived from the cross indicated. Inheritance of mutant Puc phosphatase, pucE69, a negative regulator of the JNK pathway, dominantly suppresses the severe $\operatorname{slpr}^{921}$ mutant phenotype. 
Figure 3. Mapping and cloning of the slpr locus reveal that slpr encodes a mixed lineage kinase. (A) Meiotic recombination mapping with recessive markers placed slpr to the right of singed (sn) in the 7DE region of the X chromosome. slpr complements the deletions, $D f(1) C 128$ and Df(1)HA11 (+), but fails to complement $D f(1) h l-a$ and Df(1)GE202 (-). These complementation data further refine the position of the slpr gene to the region between 7D13 and 7E2 excluding 7D187D20, which is deleted by $D f(1) H A 11$ but complemented by slpr. Next, recombination mapping with P-element transposon insertions in the region $\left({ }^{\star}\right)$ define slpr position within 7D13-7D18 (black box). A total of 2/3319 recombinant males were obtained between slpr and EP1167, whereas 0/2315 recombinants were obtained between slpr and EP1143. These results suggest that slpr is closer to the EP1143 marker, and thus likely to be found to the left of $D f(1) H A 11$. Several genes in the region $\left(T b h, f_{S}(1) h\right.$, mys, smox, Traf2, nAcRe, and Trf2) were useful landmarks to correlate the genetic and physical map with the genome sequence. (B) Among predicted and known genes in the defined region, we found a candidate gene encoding a protein kinase, CG2272. A genomic fragment encompassing CG2272 and closely neighboring CG15339, derived from the P1 clone DS08402, was used for slpr mutant rescue experiments. (C) Presence of the genomic transgene is sufficient to rescue the embryonic dorsal open phenotype of slpr. To identify the slpr mutant chromosome among the experimental population of embryonic cuticles, the slpr chromosome was marked with another recessive cuticle marker, gt. Alone, $g t / Y$ embryos show selective loss of ventral denticles; $g t, s l p r / Y$ recombinant embryos display both ventral defects and a large dorsal hole. In the presence of the genomic transgene, $P\{s l p r\}$, the dorsal open phenotype is rescued, whereas the $g t$ phenotype is unaffected. (D) An EST clone, GH26507, derived from the CG2272 locus is 5129 nucleotides, containing 8 exons (black boxes) and 7 introns (lines) relative to genomic DNA. Genbank accession no. AY045717. The cDNA encodes an ORF of 1148 amino acids (see comment in Materials and Methods) with homology to mixed lineage kinases, which display an N-terminal SH3 domain, a catalytic kinase domain, leucine zipper motifs (LZ), and a Cdc42/Rac-interacting binding motif (C). slpr alleles, 921 and 3P5, each have a mutation within the kinase domain as indicated.

each. One candidate appeared particularly relevant. This gene, CG2272, is predicted to encode a protein with sequence homology to mammalian members of the MLK family. These kinases have been implicated in JNK signal transduction at the level of the JNKKK (Rana et al. 1996; Teramoto et al. 1996; Tibbles et al. 1996). We obtained and sequenced in entirety two cDNA clones representing expressed sequence tags (ESTs) derived from this locus (Rubin et al. 2000). The larger clone, GH26507, is 5129 bp in length and contains a complete ORF of 1148 amino acids (Fig. 3D). Northern blot analysis reveals the expression of uniformly sized transcripts of $\sim 5-6 \mathrm{~kb}$ in fly embryos and adults, suggesting that the EST clone, GH26507, is likely to represent a near full- length message (data not shown). In embryos, GH26507 transcripts are expressed ubiquitously at low levels (data not shown).

Next, total RNA transcripts were prepared from handpicked embryos mutant for each slpr allele and subjected to RT-PCR amplification with GH26507-derived primers. Sequence analysis of cDNA derived from slpr mutant embryos revealed molecular lesions associated with this locus. In fact, cDNA from both slpr alleles harbored a single-point mutation within the MLKcoding region. These lesions were verified to be present in slpr mutant genomic DNA, indicating that slpr encodes Drosophila MLK. Figure 3D shows the nature and position of the mutations, which are both located 
in the kinase domain. The 921 allele produces an amino acid substitution in kinase subdomain IX (Hanks et al. 1988 ) that changes a highly conserved glutamate to lysine. The weaker allele, 3P5, produces a premature stop codon predicted to truncate the protein within the last kinase subdomain. In addition to finding mutations in the MLK gene in slpr mutant DNA and transcripts, we rescued the slpr mutant phenotype with a transgene encompassing $14 \mathrm{~kb}$ of genomic DNA, including CG2272 and the closely neighboring CG15339 gene (Fig. 3B,C). Rescue with this genomic fragment, coupled with the presence of mutations in the MLK-coding region in slpr mutant DNA, confirms that MLK is encoded by the slpr gene.

\section{The mixed lineage kinase family of JNKKKS}

MLK proteins consist of an N-terminal src-homology 3 (SH3) domain (Fig. 3D), followed by a kinase domain with key amino acids resembling the known signatures of both tyrosine and serine/threonine kinases; hence, the name mixed lineage kinase (Dorow et al. 1993; Katoh et al. 1995). Just C-terminal to the kinase domain are two leucine zipper (LZ) motifs thought to mediate dimerization (Leung and Lassam 1998; Vacratsis and Gallo 2000). Following the LZ region, there is a short stretch of amino acids with homology to Cdc42/Rac interacting binding (CRIB) motifs that may serve as a site of interaction with those small GTPases (Burbelo et al. 1995; Teramoto et al. 1996; Bock et al. 2000). The remainder of the Slpr protein displays no known motifs and has no significant homology to its mammalian counterparts. Slpr is most related to the human MLK family of three proteins with up to $56 \%$ amino acid identity within the $\mathrm{N}$-terminal region, consisting of $\sim 510$ amino acids including the kinase domain (Fig. 4) (Dorow et al. 1993, 1995; Gallo et al. 1994; Ing et al. 1994; Katoh et al. 1995). The structural features of MLK proteins have provided clues to their function and support their role as important signal transducers for the JNK pathway, conveying signals from upstream GTPases to downstream JNKKs, (Rana et al. 1996; Teramoto et al. 1996; Tibbles et al. 1996; Hirai et al. 1997; Merritt et al. 1999).

Interestingly, sequence comparisons of the kinase activation loop among MLK family members reveal that Slpr shares two critical target phosphorylation sites, Thr 287, analogous to Thr 277 in human MLK3 required for kinase autophosphorylation and Ser 291, analogous to Ser 281, targeted by members of the GCK-IV family of MKKKKs related to the fly Misshapen (Msn) protein kinase (Su et al. 1998; Dan et al. 2001; Leung and Lassam 2001). Thus, as suggested by results from mammalian studies, it is possible that Msn, Slpr, and dRacl participate in a ternary signaling complex to stimulate JNK signal transduction.

\section{Placement of slpr function in the INK pathway}

In the process of dorsal closure, dRacl appears to be a primary upstream activator of JNK signaling (Harden et
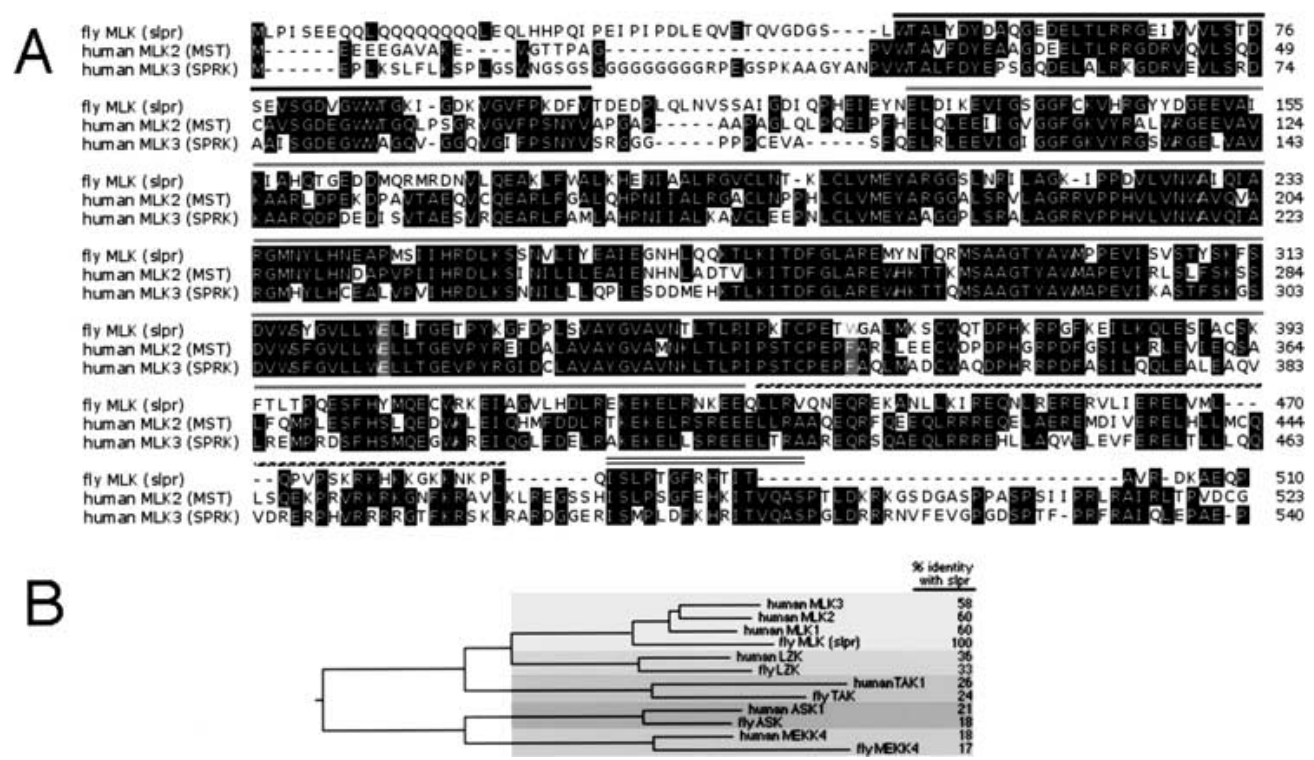

Figure 4. Slpr is related to human mixed lineage kinases among a larger group of JNKKK proteins. (A) Protein sequence alignment of fly MLK (Slpr) with human family members MLK2 (MST) and MLK3 (SPRK) in the N-terminal region encompassing the conserved domains as indicated above the sequence, SH3 (black line), kinase (gray line), LZ (dotted line), CRIB (double line). Residues highlighted in gray mark the sites in which slpr mutations are located. $(B)$ Phylogenetic relationship of Slpr compared with the putative JNKKK protein kinase group. Relationship is based on kinase sequence only, using the CLUSTAL method with PAM250 residue weight. Five of six families are represented in the fly genome as a single homolog compared with human sequences. The MLK family shares $\sim 60 \%$ amino acid identity with Slpr. The kinases with leucine zipper motifs in the LZK branch are $\sim 36 \%$ identical to Slpr. Less related kinases in the JNKKK group are TAKs ( 25\% identity), ASKs $(\sim 20 \%$ identity), and MEKKs $(\sim 18 \%$ identity), which show the greatest sequence divergence. 
al. 1995, 1999; Glise and Noselli 1997; Ricos et al. 1999). To position slpr function in the JNK pathway relative to the GTPase, we performed additional genetic epistasis tests. Due to the difficulty in following all relevant chromosomes in the embryo, we turned to the adult Drosophila eye to evaluate a possible genetic interaction. Expression of wild-type $d R a c 1$ in the eye, under regulation by the glass promotor, causes a rough, glazed appearance (Fig. 5) (Nolan et al. 1998). This phenotype is dominantly suppressed by $50 \%$ reduction in the levels of JNK signal transducers, msn, slpr, hep, and bsk. Heterozygosity at the puc locus, encoding a negative regulator of JNK signaling acting in opposition to bsk, dominantly enhances the dRac1-induced rough eye. To assess whether other putative JNKKKs in Drosophila can interact in this assay, we included Drosophila TAK (dTAK) in our analysis (Fig. 5). Unlike slpr, removal of one copy of dTAK has no effect on the dRac1-induced eye phenotype. These data suggest that there is a dosage-sensitive interaction between the JNK pathway and dRacl function in this tissue whereby increased dRacl activity can be suppressed by reduction in downstream components including slpr. Although it is not known whether $d T A K$ is expressed in the eye or at what level, $d T A K$ shows no genetic interaction with $G M R$-dRac1. Thus, slpr appears to be a relevant JNKKK in this assay. Taken together, our

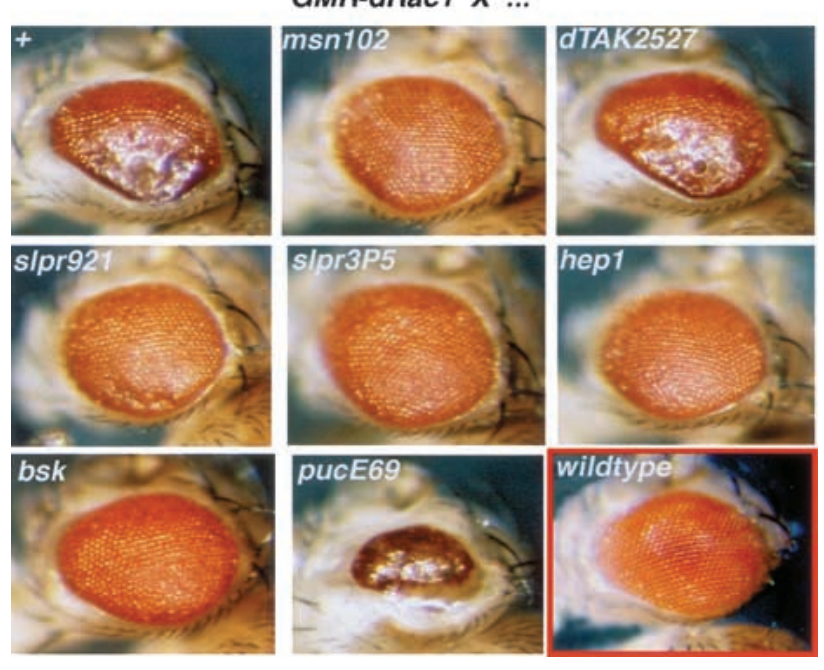

Figure 5. JNK pathway components lie genetically downstream of $d R a c 1$ in the adult eye. Expression of wild-type $d R a c 1$ under the control of the glass promotor (GMR-dRac1) in the Drosophila eye causes a rough appearance (top, left). Crossing W; GMR-dRac1 males to females that are wild type $(+)$, or mutant for msn, slpr, hep, bsk, or dTAK tests for epistatic interactions. Shown are eyes from female progeny with the indicated heterozygous mutation in addition to one copy of GMR-dRac1. Reducing the gene dosage of $m s n$, slpr, hep, and bsk by one-half significantly rescues the rough eye phenotype in comparison with $w /+G M R-d R r a c 1 /+$. A puc mutation enhances the phenotype. Notably, loss of $d T A K$ function, another putative JNKKK, does not suppress the GMR-dRac1-induced rough eye. Crosses were performed at $29^{\circ} \mathrm{C}$. A wild-type eye is shown for comparison (red box, bottom, right). epistasis tests are consistent with slpr function being required downstream of $d R a c 1$ and upstream of $b s k$ (Fig. 7, below).

\section{Role of slpr in tissue polarity}

The acquisition of polarity within the plane of an epithelium is important for the function of epithelial layers in addition to the polarity associated with the apicalbasal axis. Previous studies of epithelial planar polarity have implicated a noncanonical Frizzled pathway (Mlodzik 1999). The current model is that Fzl activates JNK activity through a pathway that includes Dishevelled (Dsh) and the RhoA GTPase. Tissue polarity mutants show disruptions in the polarized orientation of hairs and bristles as shown, for example, in Figure 6 in a $d s h^{1}$ mutant animal. To determine whether Slpr links RhoA activity to the JNK pathway, we examined the adult cuticle phenotype of slpr mutant clones in both the wing and notum, in which the tissue polarity phenotype is clearly visible. Loss of slpr activity in mutant clones was not associated with any obvious polarity defects (Fig. 6), suggesting that another JNKKK may mediate the activation of JNK activity during the establishment of planar polarity. It is unlikely that dTAK is this JNKKK, because $d T A K$ mutant animals are homozygous viable with no apparent tissue polarity defects (Vidal et al. 2001) (B. Stronach and N. Perrimon, unpubl.).

\section{Discussion}

Considerable research has shown that dorsal closure is regulated by a canonical MAP kinase-signaling module remarkably similar to the mammalian stress-signaling pathway involving a phosphorylation cascade that culminates with activation of JNK and its substrate, Jun. During dorsal closure, JNK signaling mediates gene expression, accumulation of cortical cytoskeleton, and movement of the epidermis toward the dorsal midline. Loss of signaling results in defective cell shape changes, failed closure, and lethality. Precise regulation of signaling activity in leading edge cells is necessary for proper closure, however, the identities of signals and receptors that trigger JNK activation and link membrane components to the kinase cascade are still largely unknown. Genetic identification of mutants that fail to undergo dorsal closure may uncover such components. In this study, we describe one mutant, slpr, that displays a severe dorsal open cuticle phenotype indicative of a complete failure of closure. Mutations in slpr phenocopy known JNK pathway mutants in Drosophila.

The genetic analysis of slpr described here has uncovered the identity of a missing component in our current understanding of JNK signal transduction during epithelial morphogenesis (Fig. 7). Cloning revealed that slpr encodes a mixed lineage kinase highly related to mammalian MLKs that have been shown to stimulate JNK activity when overexpressed in cell-based assays (Rana et al. 1996; Teramoto et al. 1996). Several lines of evidence 

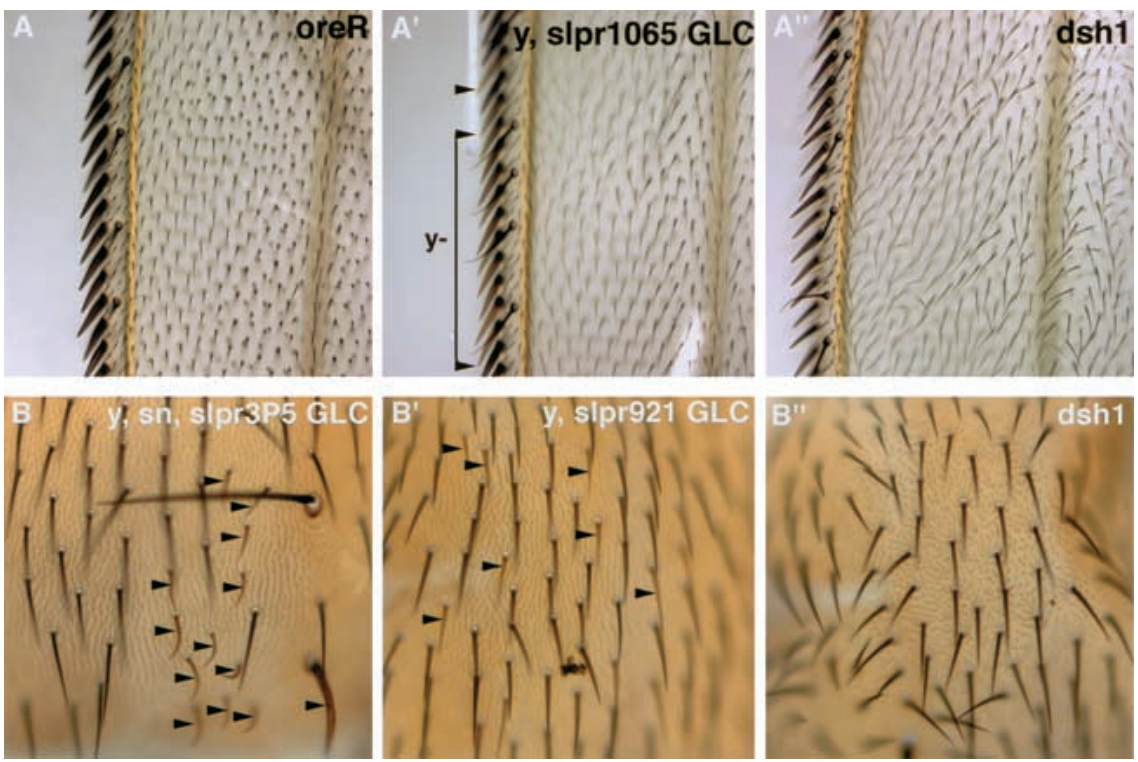

Figure 6. Loss-of-function slpr mutant clones in adult tissues have normal epithelial planar polarity. Adult wings of wild-type (oreR) flies show a uniform polarized orientation of hairs, pointing distally downward $(A)$. slpr clones marked by yellow $(y)$ are only visualized in bristles at the margin (arrowheads), yet large $y, s l p r$ clones show normal orientation of hairs neighboring the margin $\left(A^{\prime}\right)$. This is in comparison with the disrupted orientation of hairs in the $d s h^{1}$ wing $\left(A^{\prime \prime}\right)$. Planar polarity is also evident in hairs of the adult notum. Clones of slpr mutant tissue are demarcated by $y$ and singed $(s n)(B)$ or only y $\left(B^{\prime}\right)$ bristles (arrowheads). Smaller hairs in the region of the clone show normal polarized orientation, pointing posteriorly downward. In contrast, the hairs of $d s h^{1}$ mutant nota are randomly oriented, indicating a defect in planar polarity $\left(B^{\prime \prime}\right)$. indicate that slpr encodes Drosophila MLK. First, a transgenic copy of the MLK gene is sufficient to rescue slpr mutants. Second, the MLK-coding sequence is mutated in genomic DNA and cDNA from slpr mutant embryos. Loss-of-function, dorsal open slpr mutants harbor-

\begin{tabular}{|c|c|c|}
\hline & $\begin{array}{c}\text { FLY } \\
\text { DORSAL } \\
\text { CLOSURE }\end{array}$ & MAMMALS \\
\hline $\begin{array}{l}\underset{\downarrow}{\text { signal }} \\
\text { GTPase } \\
\text { MKKKK }\end{array}$ & $\begin{array}{c}? \\
\text { dRac1 } \\
\text { msn }\end{array}$ & $\begin{array}{c}\text { cytokines/stress } \\
\text { Rac/Cdc42 } \\
\text { GCK/PAK }\end{array}$ \\
\hline MKKK & slpr(MLK) & MLK/ LZK/MEKK/TAK/ASK/Tpl-2 \\
\hline $\begin{array}{c}\downarrow \\
\text { MKK } \\
\substack{\text { MAPK } \\
\downarrow} \\
\text { target }\end{array}$ & $\begin{array}{l}\text { hep } \\
\text { bsk } \\
\text { dJun }\end{array}$ & $\begin{array}{c}\text { MKK4/MKK7 } \\
\text { JNK } \\
\text { cJun/ATF2 }\end{array}$ \\
\hline & $\begin{array}{l}\text { cell sheet } \\
\text { migration }\end{array}$ & $\begin{array}{l}\text { growth, } \\
\text { differentiation, } \\
\text { apoptosis }\end{array}$ \\
\hline
\end{tabular}

Figure 7. slpr, encoding Drosophila MLK, is the MKKK for the Jun kinase pathway during dorsal closure in the fly. A canonical signaling pathway is indicated at left. Examples of mammalian proteins participating at each step are indicated at right. In Drosophila, dorsal closure involves signaling through the JNK pathway to control gene expression and cell sheet morphogenesis. Although the initiating signal is not known, it is likely that an early event is activation of dRac1, which by analogy to signal transduction with mammalian MKKKKs, participates with Msn to activate Slpr, the JNKKK. Sequential phosphorylation events through the kinase module, Slpr, Hep, and Bsk, lead to activation of dJun, regulation of gene expression, and cell sheet movement. ing molecular lesions in the kinase domain provides compelling genetic evidence that MLK proteins play a critical role in JNK signal transduction during morphogenesis, and that this role requires the kinase activity. Furthermore, our genetic epistasis data support the biochemical data that MLK proteins serve to join upstream GTPase activity to downstream AP-1 transcriptional activity. This represents the first genetic evidence that MLKs are relevant physiological regulators of JNK activity in vivo.

In Drosophila, signaling through JNK is required for a variety of processes including morphogenesis in the embryo and the adult, epithelial planar polarity, immunity, and apoptosis (Stronach and Perrimon 1999). Our data indicate that slpr is absolutely required for dorsal closure and we have not been able to detect under the experimental conditions used, an essential role for slpr in either immunity or tissue polarity. Clonal analysis of slpr in the wing and notum do not indicate a role for slpr in planar polarity of hairs; however, lack of a polarity phenotype in clones has been noted for other members of the JNK pathway. Despite this, a role for members of the JNK cascade in the establishment of planar polarity has been proposed on the basis of the ability of loss-of-function mutations to suppress a polarity phenotype associated with gain-of-function $\mathrm{Fz}$ or dsh (Boutros et al. 1998; Paricio et al. 1999; Weber et al. 2000). Together, these observations raise the possibility that a redundant function may mask a requirement for slpr in planar polarity and additional experiments will be necessary to uncover this function.

Studies on null $d T A K$ mutant flies have shown a requirement for dTAK in innate immunity to microbial infection (Vidal et al. 2001) (D. Schneider, pers. comm.). Further analysis of $d T A K$ mutant flies indicate that dTAK does not play a major role, if any, in either tissue polarity or dorsal closure, because $d T A K$ mutant flies are homozygous viable and fertile with no visible phenotype 
(Vidal et al. 2001; B. Stronach and N. Perrimon, unpubl.). Altogether, we propose that different JNKKKs are activated by different signals, and thus dedicated to different developmental processes. Further work will be needed to clarify this hypothesis. This includes the characterization of the mutant phenotypes of the other putative JNKKKs (LZK, ASK, MEKK4) and a clear demonstration that these kinases activate JNK activity in vivo. We note, for example, that dTAK has only been shown to activate JNK in overexpression assays, both in vitro and in vivo (Takatsu et al. 2000; Mihaly et al. 2001). Moreover, during an immune response, dTAK activates NFKB /Vidal et al. 2001). It has not yet been reported whether dTAK can stimulate JNK activity during immune challenge. Possibly, the specificity of these JNKKKs will reside in their abilities to activate not only the JNK pathway but also additional pathways, such as NFKB in the case of dTAK.

To date, the increasing number of mammalian protein kinases implicated as JNKKKs on the basis of sequence information and ability to activate or block JNK signaling in cell transfection or other in vitro assays underscores the potential for stunning complexity at the upper tiers of the pathway. This notion serves to reinforce and emphasize the need for thorough genetic analysis of these proteins. To date, targeted gene disruptions in the mouse have been reported only for the MEKK family, yet these studies suggest that individual MEKKs regulate unique cell behaviors including morphogenesis and apoptosis (Yujiri et al. 1998, 2000; Garrington et al. 2000; Yang et al. 2000). By taking advantage of the reduced gene redundancy in Drosophila relative to mammalian systems and the various defined processes in which JNK signaling is required, we are poised to answer questions of specificity at the level of cellular and developmental responses as well as at the level of upstream and downstream partners.

\section{Materials and methods}

\section{Genetics}

Germ-line clones of slpr were generated by crossing $O v O^{D 1}$ $F R T^{101} / Y$; hs-Flp38 males to y w mutant $v$ FRT $T^{101} / F M 7$ females. Third instar larval progeny were placed at $37^{\circ} \mathrm{C}$ for $2 \mathrm{~h}$ each day for $3 \mathrm{~d}$. Heat-treated, non-FM7 females with germ-line clones were mated with wild-type males and allowed to lay eggs. A total of $50 \%$ of the fertilized eggs failed to hatch and showed a severe dorsal open phenotype. The remainder of embryos hatched and developed into adult females, showing that the paternally contributed copy of the mutant gene can functionally rescue the loss of maternal product. Heat-treated females with germ-line clones also have somatic clones in tissue derived from the larval imaginal discs. Clones in the wings and nota were analyzed for tissue polarity defects.

$s l p r^{3 P 5} / Y$ hemizygous embryos derived from heterozygous females die during embryogenesis or larval stages. Dead embryos exhibit a variable dorsal open phenotype. Embryos derived from females with homozygous $\operatorname{slpr}^{3 P 5}$ germ-line clones, which do not receive a wild-type slpr gene from their father, develop large anterior dorsal holes, indicative of severe defects in dorsal clo- sure. All slpr ${ }^{921} / Y$ hemizygous embryos derived from heterozygous females die during embryogenesis with a severe dorsal open cuticle phenotype. Embryos derived from females with slpr ${ }^{921}$ germ-line clones, which are not paternally rescued, die with a severe cuticle identical to the zygotic phenotype.

The fly stocks used to map the slpr locus are described (Rorth 1996; Flybase 1999). The X chromosome visible marker stock used for meiotic recombination mapping was sc ec cv ct $v g f$. The deficiency stocks, described in Flybase (flybase.bio.indiana.edu/aberrations), were obtained from the Bloomington or Umea stock centers. Males bearing a deficiency covered by a duplication were crossed to slpr mutant females for complementation testing. P-element transposons used for recombination mapping were obtained from the Bloomington Stock Center and are described in Flybase (flybase.bio.indiana.edu/transposons). They are viable EP lines that contain a white marker. $^{+}$ Males with the $\mathrm{P}$ element $\left(w, p E P\left[w^{+}\right] / Y\right)$ were crossed to slpr mutant females (y w slpr $\left.{ }^{921} F R T^{101}\left[W^{+}\right] / F M 7\right)$. Heterozygous female progeny were collected and crossed to wild-type males. The total number of male progeny from this cross was counted. Among those, we scored the number of viable $w$ males, which had neither the lethal slpr mutation nor the $w^{+} \mathrm{P}$ element.

Other stocks used in this study include $d T A K^{2527}$ (kindly provided by D. Schneider, Stanford University, CA), $\mathrm{msn}^{102}$, hep ${ }^{1}$, $b_{s k}{ }^{1}$, puc $^{E 69}$, slpr ${ }^{1065}$ (a severe allele for which the molecular nature is unknown), and c/un ${ }^{a s p}$ under the control of a heatinducible promotor (Treier et al. 1995). Under the conditions used herein (described in the Figure 2 legend), we rarely detect defects in heat-treated embryos from this stock. Those embryos with defects $(<10 \%)$ have very small anterior holes. When HSclun $^{\text {asp }}$ is crossed with slpr mutants and embryos are heat shocked, a new class of cuticle phenotypes is observed that displays dorsal holes less severe than the slpr mutants alone, but more severe than the defects observed with HS- cJun ${ }^{a s p}$ alone. These are presumed to be the class of partially rescued mutant embryos.

\section{Molecular biology}

PCR amplification of deficiency DNA was carried out on embryonic genomic DNA. Deficiencies were balanced with a green fluorescent protein (GFP)-positive chromosome. Lack of GFP fluorescence allowed identification of $D f / Y$ embryos. Twenty embryos were crushed in $100 \mu \mathrm{L}$ of buffer $(10 \mathrm{mM}$ Tris at $\mathrm{pH} 8.2$, $1 \mathrm{mM}$ EDTA, $25 \mathrm{mM} \mathrm{NaCl}, 200 \mu \mathrm{g} / \mathrm{mL}$ Proteinase K). The homogenate was incubated at $37^{\circ} \mathrm{C}$ for $30 \mathrm{~min}$, heated to $95^{\circ} \mathrm{C}$ for $5 \mathrm{~min}$, then frozen. We used $1-2 \mu \mathrm{L}$ of this homogenate as template for $30-\mu \mathrm{L}$ PCR reactions.

RT-PCR to identify mutations in slpr cDNA was performed. slpr germ-line clone females were crossed to males with an $\mathrm{X}$ chromosome carrying GFP. Embryos were collected and aged 20 h. Non-GFP embryos were handpicked into Ultraspec RNA reagent (Biotecx Laboratories, Houston, TX). Total RNA was prepared according to the manufacturer and used as a template for reverse transcription and PCR amplification using the Superscript One-Step RT-PCR System (GIBCO BRL Life Technologies) according to the instructions. Several PCR reactions for each allele were pooled. Amplification products were purified and cloned into the pGEM-T Easy Vector (Promega) for sequencing. Several clones per allele were sequenced on both strands to determine the presence of mutations. If mutations were identified, PCR amplification, cloning, and sequencing of genomic DNA was performed to confirm the mutation.

The longest ORF encoded by EST GH26507 is 1161 amino acids, however, the start codon does not conform well to the consensus. Another start codon is observed 13 amino acids 
downstream that would encode an ORF of 1148 amino acids. This ATG is surrounded by nucleotides that conform well to the consensus and is likely to be the true start codon.

We generated the slpr genomic rescue construct by isolating a HindIII fragment of $\sim 14 \mathrm{~kb}$ from the P1 clone DS08402, including the $M L K$ gene, and a neighboring annotated gene CG15339. This fragment was cloned transiently into pBluescript (Stratagene) and then moved into pCaSpeR 4 vector for subsequent purification and germ-line transformation. All slpr alleles, 3P5, 921 , and 1065 can be rescued to adulthood with the presence of one copy of the genomic transgene.

\section{Staining and imaging}

In situ hybridization to embryos was performed as described (Stronach et al. 1996). The $d p p$ antisense riboprobe, corresponding to $d p p$ coding sequence, was generated by use of the Boehringer Mannheim Genius RNA labeling kit according to the manufacturer. Stained embryos were mounted in $70 \%$ glycerol. Images were captured with the SPOT digital camera (Diagnostic Instruments) using differential interference contrast (DIC) optics on a Zeiss Axiophot microscope. Immunofluorescence was performed as described (Patel 1994). Mouse anti-fasciclin III (7G10) was used at 1:40 (Patel et al. 1987). Fluorescent images were captured with a confocal microscope system (Leica TCSNT) and were processed with Adobe Photoshop. For clonal analysis of slpr in adult tissues, wings were removed and mounted on flat slides in Euparal mountant. Nota were dissected from adults and mounted in Hoyers Mounting Medium in a depression slide. DIC images were captured as described above.

\section{Acknowledgments}

We thank D. Schneider, L. Kockel, M. Mlodzik, J. Settleman, S. Noselli, A. Martinez-Arias, and the Bloomington and Umea Drosophila Stock Centers for fly strains, the Harvard Medical School Biopolymers Facility for sequence analysis, J. Kopinja for embryo injection, and S. Cherry for critical comments on the manuscript. We also give special thanks to M. Zeidler for suggesting the name slipper. B.S. is supported by the NIH (GM19775) and N.P. is a Howard Hughes Investigator.

The publication costs of this article were defrayed in part by payment of page charges. This article must therefore be hereby marked "advertisement" in accordance with 18 USC section 1734 solely to indicate this fact.

\section{References}

Adams, M.D., Celniker, S.E., Holt, R.A., Evans, C.A., Gocayne, J.D., Amanatides, P.G., Scherer, S.E., Li, P.W., Hoskins, R.A., Galle, R.F., et al. 2000. The genome sequence of Drosophila melanogaster. Science 287: 2185-2195.

Bock, B.C., Vacratsis, P.O., Qamirani, E., and Gallo, K.A. 2000. Cdc42-induced activation of the mixed-lineage kinase SPRK in vivo. Requirement of the Cdc42/Rac interactive binding motif and changes in phosphorylation. J. Biol. Chem. 275: 14231-14241.

Boutros, M., Paricio, N., Strutt, D.I., and Mlodzik, M. 1998. Dishevelled activates JNK and discriminates between JNK pathways in planar polarity and wingless signaling. Cell 94: 109-118.

Burbelo, P.D., Drechsel, D., and Hall, A. 1995. A conserved binding motif defines numerous candidate target proteins for both Cdc42 and Rac GTPases. J. Biol. Chem. 270: 2907129074.
Campos-Ortega, J.A. and Hartenstein, V. 1997. The embryonic development of Drosophila melanogaster. Springer, Berlin/ New York.

Chang, L. and Karin, M. 2001. Mammalian MAP kinase signalling cascades. Nature 410: 37-40.

Chou, T.B. and Perrimon, N. 1996. The autosomal FLP-DFS technique for generating germline mosaics in Drosophila melanogaster. Genetics 144: 1673-1679.

Dan, I., Watanabe, N.M., and Kusumi, A. 2001. The Ste20 group kinases as regulators of MAP kinase cascades. Trends Cell. Biol. 11: 220-230.

Davis, R.J. 2000. Signal transduction by the JNK group of MAP kinases. Cell 103: 239-252.

Dorow, D.S., Devereux, L., Dietzsch, E., and De Kretser, T. 1993. Identification of a new family of human epithelial protein kinases containing two leucine/isoleucine-zipper domains. Eur. J. Biochem. 213: 701-710.

Dorow, D.S., Devereux, L., Tu, G.F., Price, G., Nicholl, J.K., Sutherland, G.R., and Simpson, R.J. 1995. Complete nucleotide sequence, expression, and chromosomal localisation of human mixed-lineage kinase 2. Eur. J. Biochem. 234: 492-500.

Flybase. 1999. The FlyBase database of the Drosophila genome projects and community literature. Nucleic Acids Res. 27: 85-88.

Gallo, K.A., Mark, M.R., Scadden, D.T., Wang, Z., Gu, Q., and Godowski, P.J. 1994. Identification and characterization of SPRK, a novel src-homology 3 domain-containing prolinerich kinase with serine/threonine kinase activity. J. Biol. Chem. 269: 15092-15100.

Garrington, T.P., Ishizuka, T., Papst, P.J., Chayama, K., Webb, S., Yujiri, T., Sun, W., Sather, S., Russell, D.M., Gibson, S.B., et al. 2000. MEKK2 gene disruption causes loss of cytokine production in esponse to IgE and c-Kit ligand stimulation of ES cell-derived mast cells. EMBO J. 19: 5387-5395.

Glise, B. and Noselli, S. 1997. Coupling of Jun amino-terminal kinase and Decapentaplegic signaling pathways in Drosophila morphogenesis. Genes \& Dev. 11: 1738-1747.

Glise, B., Bourbon, H., and Noselli, S. 1995. hemipterous encodes a novel Drosophila MAP kinase kinase, required for epithelial cell sheet movement. Cell 83: 451-461.

Hanks, S.K., Quinn, A.M., and Hunter, T. 1988. The protein kinase family: conserved features and deduced phylogeny of the catalytic domains. Science 241: 42-52.

Harden, N., Loh, H.Y., Chia, W., and Lim, L. 1995. A dominant inhibitory version of the small GTP-binding protein Rac disrupts cytoskeletal structures and inhibits developmental cell shape changes in Drosophila. Development 121: $903-$ 914.

Harden, N., Ricos, M., Ong, Y.M., Chia, W., and Lim, L. 1999. Participation of small GTPases in dorsal closure of the Drosophila embryo: Distinct roles for Rho subfamily proteins in epithelial morphogenesis. J. Cell Sci. 112: 273-284.

Hartkamp, J., Troppmair, J., and Rapp, U.R. 1999. The JNK/ SAPK activator mixed lineage kinase 3 (MLK3) transforms NIH $3 \mathrm{~T} 3$ cells in a MEK-dependent fashion. Cancer Res. 59: 2195-2202.

Hirai, S., Katoh, M., Terada, M., Kyriakis, J.M., Zon, L.I., Rana, A., Avruch, J., and Ohno, S. 1997. MST/MLK2, a member of the mixed lineage kinase family, directly phosphorylates and activates SEK1, an activator of c-Jun N-terminal kinase/ stress-activated protein kinase. J. Biol. Chem. 272: 1516715173.

Holland, P.M., Suzanne, M., Campbell, J.S., Noselli, S., and Cooper, J.A. 1997. MKK7 is a stress-activated mitogen-activated protein kinase kinase functionally related to hemipterous. J. Biol. Chem. 272: 24994-24998. 
Hou, X.S., Goldstein, E.S., and Perrimon, N. 1997. Drosophila Jun relays the Jun amino-terminal kinase signal transduction pathway to the Decapentaplegic signal transduction pathway in regulating epithelial cell sheet movement. Genes \& Dev. 11: 1728-1737.

Ing, Y.L., Leung, I.W., Heng, H.H., Tsui, L.C., and Lassam, N.J. 1994. MLK-3: Identification of a widely-expressed protein kinase bearing an $\mathrm{SH} 3$ domain and a leucine zipper-basic region domain. Oncogene 9: 1745-1750.

Ip, Y.T. and Davis, R.J. 1998. Signal transduction by the c-Jun $\mathrm{N}$-terminal kinase (JNK)-from inflammation to development. Curr. Opin. Cell. Biol. 10: 205-219.

Jochum, W., Passegue, E., and Wagner, E.F. 2001. AP-1 in mouse development and tumorigenesis. Oncogene 20: 2401-2412.

Katoh, M., Hirai, M., Sugimura, T., and Terada, M. 1995. Cloning and characterization of MST, a novel (putative) serine/ threonine kinase with $\mathrm{SH} 3$ domain. Oncogene 10: $1447-1451$.

Knust, E. 1997. Drosophila morphogenesis: Movements behind the edge. Curr. Biol. 7: R558-R561.

Kockel, L., Zeitlinger, J., Staszewski, L.M., Mlodzik, M., and Bohmann, D. 1997a. Jun in Drosophila development: Redundant and nonredundant functions and regulation by two MAPK signal transduction pathways. Genes \& Dev. 11: $1748-1758$.

- 1997b. Jun in Drosophila development: Redundant and nonredundant functions and regulation by two MAPK signal transduction pathways. Genes \& Dev. 11: 1748-1758.

Leung, I.W. and Lassam, N. 1998. Dimerization via tandem leucine zippers is essential for the activation of the mitogenactivated protein kinase kinase kinase, MLK-3. I. Biol. Chem. 273: 32408-32415.

- 2001. The kinase activation loop is the key to mixed lineage kinase-3 activation via both autophosphorylation and hematopoietic progenitor kinase 1 phosphorylation. I. Biol. Chem. 276: 1961-1967.

Martin-Blanco, E., Gampel, A., Ring, J., Virdee, K., Kirov, N., Tolkovsky, A.M., and Martinez-Arias, A. 1998. puckered encodes a phosphatase that mediates a feedback loop regulating JNK activity during dorsal closure in Drosophila. Genes \& Dev. 12: $557-570$.

Merritt, S.E., Mata, M., Nihalani, D., Zhu, C., Hu, X., and Holzman, L.B. 1999. The mixed lineage kinase DLK utilizes MKK7 and not MKK4 as substrate. I. Biol. Chem. 274: 10195-10202.

Mihaly, J., Kockel, L., Gaengel, K., Weber, U., Bohmann, D., and Mlodzik, M. 2001. The role of the Drosophila TAK homologue dTAK during development. Mech. Dev. 102: 67-79.

Mlodzik, M. 1999. Planar polarity in the Drosophila eye: A multifaceted view of signaling specificity and cross-talk. EMBO J. 18: 6873-6879.

Nolan, K.M., Barrett, K., Lu, Y., Hu, K.Q., Vincent, S., and Settleman, J. 1998. Myoblast city, the Drosophila homolog of DOCK180/CED-5, is required in a Rac signaling pathway utilized for multiple developmental processes. Genes \& Dev. 12: 3337-3342.

Noselli, S. 1998. JNK signaling and morphogenesis in Drosophila. Trends Genet. 14: 33-38.

Paricio, N., Feiguin, F., Boutros, M., Eaton, S., and Mlodzik, M. 1999. The Drosophila STE20-like kinase misshapen is required downstream of the Frizzled receptor in planar polarity signaling. EMBO J. 18A: 4669-4678.

Patel, N.H. 1994. Imaging neuronal subsets and other cell types in whole-mount Drosophila embryos and larvae using antibody probes. In Drosophila melanogaster: Practical uses in cell and molecular biology (ed. L.S.B. Goldstein and E.A. Fyrberg), pp. 446-487. Academic Press, San Diego, CA.

Patel, N.H., Snow, P.M., and Goodman, C.S. 1987. Characterization and cloning of fasciclin III: A glycoprotein expressed on a subset of neurons and axon pathways in Drosophila. Cell 48: 975-988.

Perrimon, N., Engstrom, L., and Mahowald, A.P. 1989. Zygotic lethals with specific maternal effect phenotypes in Drosophila melanogaster. I. Loci on the X chromosome. Genetics 121: 333-352.

Rana, A., Gallo, K., Godowski, P., Hirai, S., Ohno, S., Zon, L., Kyriakis, J.M., and Avruch, J. 1996. The mixed lineage kinase SPRK phosphorylates and activates the stress-activated protein kinase activator, SEK-1. J. Biol. Chem. 271: 1902519028.

Ricos, M.G., Harden, N., Sem, K.P., Lim, L., and Chia, W. 1999. Dcdc42 acts in TGF- $\beta$ signaling during Drosophila morphogenesis: Distinct roles for the Drac1/JNK and Dcde42/TGF- $\beta$ cascades in cytoskeletal regulation. J. Cell Sci. 112: 1225-1235.

Riesgo-Escovar, J.R. and Hafen, E. 1997. Drosophila Jun kinase regulates expression of decapentaplegic via the ETS-domain protein Aop and the AP-1 transcription factor DJun during dorsal closure. Genes \& Dev. 11: 1717-1727.

Riesgo-Escovar, J.R., Jenni, M., Fritz, A., and Hafen, E. 1996. The Drosophila Jun-N-terminal kinase is required for cell morphogenesis but not for DJun-dependent cell fate specification in the eye. Genes \& Dev. 10: 2759-2768.

Rorth, P. 1996. A modular misexpression screen in Drosophila detecting tissue-specific phenotypes. Proc. Natl. Acad. Sci. 93: $12418-12422$.

Rubin, G.M., Hong, L., Brokstein, P., Evans-Holm, M., Frise, E., Stapleton, M., and Harvey, D.A. 2000. A Drosophila complementary DNA resource. Science 287: 2222-2224.

Sluss, H.K., Han, Z., Barrett, T., Davis, R.J., and Ip, Y.T. 1996. A JNK signal transduction pathway that mediates morphogenesis and an immune response in Drosophila. Genes \& Dev. 10: $2745-2758$.

Stronach, B.E. and Perrimon, N. 1999. Stress signaling in Drosophila. Oncogene 18: 6172-6182.

Stronach, B.E., Siegrist, S.E., and Beckerle, M.C. 1996. Two muscle-specific LIM proteins in Drosophila. I. Cell Biol. 134: 1179-1195.

Su, Y.C., Treisman, J.E., and Skolnik, E.Y. 1998. The Drosophila Ste20-related kinase misshapen is required for embryonic dorsal closure and acts through a JNK MAPK module on an evolutionarily conserved signaling pathway. Genes \& Dev. 12: 2371-2380.

Takatsu, Y., Nakamura, M., Stapleton, M., Danos, M.C., Matsumoto, K., O'Connor, M.B., Shibuya, H., and Ueno, N. 2000. TAK1 participates in c-Jun $\mathrm{N}$-terminal kinase signaling during Drosophila development. Mol. Cell. Biol. 20: 3015-3026.

Teramoto, H., Coso, O.A., Miyata, H., Igishi, T., Miki, T., and Gutkind, J.S. 1996. Signaling from the small GTP-binding proteins Rac1 and Cdc42 to the c-Jun $\mathrm{N}$-terminal kinase/ stress-activated protein kinase pathway. A role for mixed lineage kinase 3/protein-tyrosine kinase 1, a novel member of the mixed lineage kinase family. J. Biol. Chem. 271: 27225-27228.

Tibbles, L.A., Ing, Y.L., Kiefer, F., Chan, J., Iscove, N., Woodgett, J.R., and Lassam, N.J. 1996. MLK-3 activates the SAPK/JNK and p38/RK pathways via SEK1 and MKK3/6. EMBO $I$. 15: 7026-7035.

Treier, M., Bohmann, D., and Mlodzik, M. 1995. JUN cooperates with the ETS domain protein pointed to induce photoreceptor R7 fate in the Drosophila eye. Cell 83: 753-760. 
Vacratsis, P.O. and Gallo, K.A. 2000. Zipper-mediated oligomerization of the mixed lineage kinase SPRK/MLK-3 is not required for its activation by the GTPase cdc 42 but is necessary for its activation of the JNK pathway. Monomeric SPRK L410P does not catalyze the activating phosphorylation of Thr258 of murine mitogen-activated protein kinase kinase 4 . J. Biol. Chem. 275: 27893-27900.

Vidal, S., Khush, R.S., Leulier, F., Tzou, P., Nakamura, M., and Lemaitre, B. 2001. Mutations in the Drosophila dTAK1 gene reveal a conserved function for MAPKKKs in the control of rel/NF-кB-dependent innate immune responses. Genes \& Dev. 15: 1900-1912.

Vogt, P.K. 2001. Jun, the oncoprotein. Oncogene 20: 2365-2377.

Weber, U., Paricio, N., and Mlodzik, M. 2000. Jun mediates Frizzled-induced R3/R4 cell fate distinction and planar polarity determination in the Drosophila eye. Development 127: 3619-3629.

Yang, J., Boerm, M., McCarty, M., Bucana, C., Fidler, I.J., Zhuang, Y., and Su, B. 2000. Mekk3 is essential for early embryonic cardiovascular development. Nat. Genet. 24: 309-313.

Yujiri, T., Sather, S., Fanger, G.R., and Johnson, G.L. 1998. Role of MEKK1 in cell survival and activation of JNK and ERK pathways defined by targeted gene disruption. Science 282: 1911-1914.

Yujiri, T., Ware, M., Widmann, C., Oyer, R., Russell, D., Chan, E., Zaitsu, Y., Clarke, P., Tyler, K., Oka, Y., et al. 2000. MEK kinase 1 gene disruption alters cell migration and c-Jun $\mathrm{NH} 2$ - terminal kinase regulation but does not cause a measurable defect in NF-к B activation. Proc. Natl. Acad. Sci. 97: 7272-7277.

Zeitlinger, J., Kockel, L., Peverali, F.A., Jackson, D.B., Mlodzik, M., and Bohmann, D. 1997. Defective dorsal closure and loss of epidermal decapentaplegic expression in Drosophila fos mutants. EMBO I. 16: 7393-7401. 


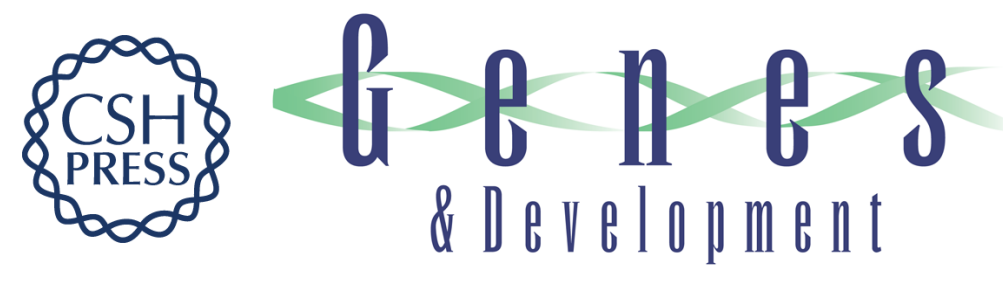

\section{Activation of the JNK pathway during dorsal closure in Drosophila requires the mixed lineage kinase, slipper}

Beth Stronach and Norbert Perrimon

Genes Dev. 2002, 16:

Access the most recent version at doi:10.1101/gad.953002

References This article cites 61 articles, 40 of which can be accessed free at: http://genesdev.cshlp.org/content/16/3/377.full.htmI\#ref-list-1

License

Email Alerting

Receive free email alerts when new articles cite this article - sign up in the box at the top Service right corner of the article or click here.

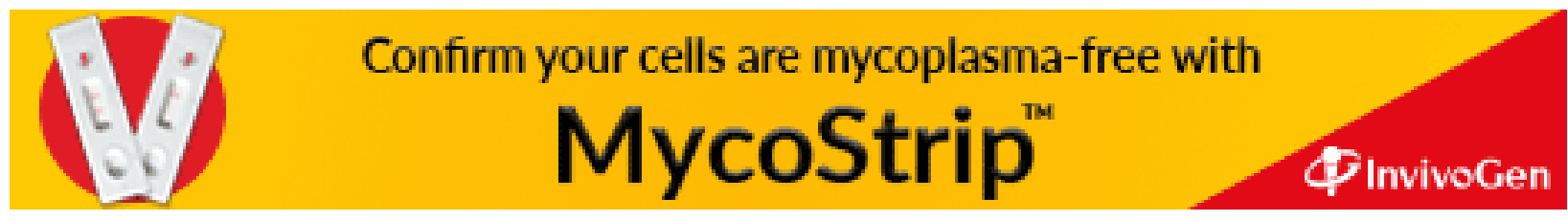

